\title{
LA RINO-FARINGITIS DEL LACTANTE
}

\author{
$\mathrm{Por}$ \\ J. SCHWARZENBERG L. $\%$ SAMUEL COSTA \\ Jafe de Setvicio \\ Ayudanic

\section{(Continuación)}

Para la apreciación de los sintomas generales que nro. voca la rino-faringitis en el lactante se presenta en el Asilo (Casa Nacional del Niño) y en el Hospital conectado con el (Hospital de Niños "Luis Calvo Mackenna"), un matrial especialmente favorable, por el control continuado que puede hacerse durante un largo tiempo de las curvas de temp?ratura. peso y crecimiento y por la obscrvación permanente a que pueden scmetrse las alteraciones del estado general y local de los niños sanos y enfermos. Muchos sindromas, que observados bajo condiciones distintas, no pasan de ser más que incidentes pasajeros $y$ de poca o ninguna importancia adquie$r \in n$ aquí un cariz totalmente diverso, cuando se comprueba como se repiten. Se prolongan en lin estado de semi latencia y se exacerban o se entrelazan los unos con los otros y cuando se ve también cómo sa van estableciendo defectos locales y ge. netales a consecuencia de ellos. Enfocado el problema de la tino-faringitis desde este punto de vista y aunque no podemos D.zar que muchas de nuestras observaciones presentan todavía grandes defectos en el registro meticuloso de los detalles, es indudable que del estudio de ellas, sobre todo de las que se han Ilevade con toda escrupulosidad. se desprenden hechos y conclusiones de sumo interés.

Si se mira la rino-faringitis como un incidante infeccioso aislado, el estudio de las curvas térmicas nos revela para muchos enfermos un alza febril más o menos acentuada $y$ 
que no dura más que 24-48 horas; para otros, estados febriles de intensidad variable también y de 3-8 días de duración con una fiebre continua o remitente y para un tercer grupo. temperaturas elevadas, que suelen prolongarse durante semanas. La a tura de la fiebre y su duración no guardan relación con la intensidad, ni con la extensión del proceso local: ciertas exacerbaciones en la curva o la reaparición de un aiza febril, suele ser, en cambio, el indici de alguna complicación. Decimcs. suele ser, porque son muchas las observaciones en las que esta coincidencia no se encuentra. El estado psíquico y organico de los niños puede mantenerse tan sin alteracion, que el alza febril constituye una sorpresa: otras veces va acompañada de llanto continuo, intranquilidad, inapetencia y decaimiento. Por lo demás, como ya lo dijimos al ocuparnos de la sintoma. tología local, no es raro que ésta falte en la iniciaciórs y que se la encuentre solamente 2-3 día después de la primera alza térmica c aun después de la notmalización dz la temperatura. Para el que no conozca o no quitra aceptar esta peculiaridad en la evolución de la rino-faringitis. tendrán que quedar en la penumbra y con un gran signe interrogativo, muchos estados trbriles del niño pequeño. Cierto es que muchas veces una tecidiva a breve plazo, permite hacer un diagnóstico retrospectivo porque se prisentan entonces síntomas locales evidentes. La efectividad de este hecho se comprueba en un número bastanie alto de nuestras observacicnes.

$\mathrm{Si}$ aquelio vale para los lactantes tomades en general, en los premaruros, en cierto grupo de recién nacidos. en distróficos muy acentuados y en algunos diatésiccs. la reacción febril frente a la infección rino-faringea falta muchas veces y aun suete ser remplazada por verdaderas hipctermias, tarto más graves, cuanto más intenso es el compromiso del estado general del enfermo. En estos casos la fiebre puede apazecer después de dos o más días - hemos visto prolongarse estas hipotermias hasta 15 dias-, cuando el pequeño organismo ha logrado hacer la reversión de su ectuilibrio orgánico, funcional y humcral. que el primer ataque infeccioso habia quebrantado. Puede presentarse también 1-3 días antes de la muerte, sea que hayan sobrevenido complicaciones de carácter septisémico de mayor gravedad o no. Cuando esto no sucede. la temperatura tiende a normalizarse paulatinamente, de acuerdo con la mejoría de los dimás síntomas, que se presentan en cada caso. Por 10 demás, vemos que en este tipo de iactantes las temperaturas sub-febriles pueden mantenerso durante semanas y meses después de terminada la fase aguda de cu 
rino-faringitis. Esta curva no se ve interrumpida más que por alteraciones que corresponden a alguna incidencia aguda intercurrente, semejante en todo a las que presentan los demás lactantes tambièn. Recalcaremos que la mayoria de los pequeños enfermos que revelan esta tendencia a la hipotermia pertenecen al grupo que presenta las alteraciones respiratorias. que ana!izamos anteriormente, o sea, la apnea, la respiración superficial, la cianosis. etc, y el meteorismo, con to cual se manifiesta, a nuestra manera de ver, un compromiso encéfaio buibar o, por 'o menos, del sistema neuro-vegetativo que, I su $v i z$, puede explicar una anarquía o falla de la regulación caló:ica. Notorio es, además, que algunos de estos niños présentan signos encefálicos francos lestrabismo, nistagmus, estados convulsivos pasajeros, etc.).

La incidencia aguda de 1 a rino-faringitis en el lactante. cuando se enfoca aisladaminte, presenta en su curva térmica las caracteristicas señaladas; pero el problema cambia fundamentzlmerite si se analizan los registros de temperatura de aque!los niños que han permancido durante un largo tiempos en e! Servicio. Miradas en conjunto, llama la atención, desciz luego, la frecuencia, mayor en il primer semestre y más alejada desfués, con que figuran en cilas los diagnósticos de riniis $y$ rinc-faringitis, en correstondencia casi siempre con alzas febriles, crda una de las cuales presenta los caracters que bemos indicado como típicos del ataque agudo rino-farírgeo. Sin embargo, en cada curva, en total aparecen ciertas modalidades que es interesante añar. Pueden separarse numercsas observaciones. generalnote de niños mayores de 3 meses, en las que se ven aparec?" periódicamente con int?rvalos de dias o semanas. alzas de timperatura has:a 39 o más grados de uno o más dias de furación y qua no tienen otr.l explicación que la que da la sintomatología de una rino-faringitis; en los espocios libres se comprueba siemore una temperatura nermal. En otros enfermos estos pariodos febribs son de nivel más bajo y de evo!ución más arrastrada 115 ó más días de duración). Pero hay un grupo de lactantes y se trata gereralmente de los que pirsentan sus primeros sintomas antes del mes de edad, en los cuales un control cuidadoso de $\mathrm{i}$ temperatura revela que entre una y otra de esas irrupcienes ficbriles, no se obtiene, salvo uno que otro dia, una normaliza. ción de la temperatura: se mantione en ellos una curva por sncima de $37,5^{\circ}$, cuya única justificación se encuentra en 12 per- ietencia de pequeños signos rino-faríngsos. A medida que $\therefore$ paciente se acerca o sobrepasa el año de edad, se van acentur:- 
do y prolongando generalmente los periodos afebriles, hasta desaparecer por completo, junto con la predisposición a la repetición de los catarres del rino-farinx. Las curvas de los prematuros, distróficos, raquíticos y diatésicos, nos muestran las mismas caracteristicas que las d? este último grupo: pero se distinguen por una mayot frecuencia y una mayor gravedad de las complicaciones y una repeccusión mucho más manifiesta sobre el desarrollo y el estado general de los niños. Mientras menores sean. estos enfermitos o mientras mayor sea el compremiso orgánico-humoral do ellos, tanto mayor tes todavía en ellos la tendencia a hipotermias pasajeras, sobre todo frente a alguna exacerbación del proceso inflamatorio local.

Es indudabie, pues, que el análisis de la curva térmica nos da indicaciones de un alto valer cignóstico: pero no es menos interesante, desde este punto de vista, el estudio de la curva fonderal, cuya apreciación real hasta en sus más tinos detalles sólo puede baczrse en las observaciones de un servicio clínico. Aquí vemos al lado de niños, cuvo ascenco pondorai no sufre la más leve alteración y que soportan una rino-faringitis con un mínimo de mclestias o de menifestaciones generales, ctros que presentan una detención del peso o bajas apreciables. cuya duración fluctúa entre poccs dís y una semana o más. La gran mayoría de los pequeñcs enfermos pertenece a este úl. timo grupo. En ciertos casos. la causa para estas anomalías de la curva ponderal se encuentra en la inapetencia, en el rechazo de la alimentación y tamtién en las dificultades qua suelen presentarse en la deglución. Otras veces son la expresión de un trastorno nutritivo agudo sobre agregado (dispspsia, toxiccsis, etc. ), de una deshidratación interna o de una sed relativa. Y no faltan casos en los cuales no son más que Ia manifestación de un estado constitucional especial (hidrolatilidad, diatesis exudativa, neuropática, etc.). Sabide es, por Jo demás, que ellas se presentan como acompañantes corrientes de cualquiera infección aguda en el lactante. Interesan: para el caso de la rino-faringitis es que esa sintomatología aparatosa induce a ignorar el origen para-enteral verdadero y ica: de ella. Jo que no acontece en circunstancias idénticas para n? caso de una neumenía pielitis. meningitis, ctc. cayo sindroma específico se impone con más facilidad que el de aquell. Algo análogo sucede también con la otitis media aguda. aungus en la gran maycría de los casos, ella no es más que una extensión de la inflamación rino-faríngea al oido medic: pero su sintomatología es generalmente más impresionante. tanto obic tiva como subjetivamente. El diegróstico de la infacción parn- 
enteral se hace a esa altura sin dificultad; pero se pasa por alto tc:do el períodc inicial, o sea, sul Localización rino-faríngea.

Lo cotriente es que la baja de peso o la simple detención de la curva coincida con la iniciarión del alza de la temperatura o con la aparición de los prinures sintomas de la rinofaringitie; perc son muchos los gráficos en que se observa un descenso pondira? progresivo durerte varios días antes aue se encuentren aquéllos o antes qua se manifieste ia fiebre. En otres se ve que los síntomas locales van acompañados nada más que de esta pérdida $\mathrm{c}$ detención del peso y de una reducción de la alimentación, sin que la temperatura se haya elevado $y$ sin que el estado general se afiete mayormente. En estos casos uele presentarse uno que otro vómito aislado y a distintas horas durante los días criticos; se observan alteraciones del carácter de las deposiciones, perc con menos frecuencia. Tal como lo expusimos en lo que se refiere a la fiebre. los sintomas locales rino-faríngeos también pueden manifestarse solamente cuando la caída del paso va se ha detenido o cuando la curva ya ha comenzado a ascender nuevamente.

En los prematuros, recién nacidos, que más tarde se revelan comc portadores de alguna diátesis y en los distróficos. se $\checkmark \varepsilon$ cue aun con una alimentación natural, los descensos de pesc o una curva ponderal apenas horizontal, pueden mantencte durante algunas semanas, como acomptñantes de una sintcmatolegía rino-faríngea inciscutible, con compromiso manifiesto del estado general o sirn él. En forma muy parecida reaccionan los niños que han estado sometidos a cualquier tégimen defectuoso, sea que su alimentación haya estado rerargada de grasas, hidratos de carhono, etc., o que se haya mantenido en ella un déficit prolongado de cualesquiera de los elementos nutritivos esenciales. Además, el trastorno nutritivo agudo concomitante se desencadena en estos enfermos con mayor facilidad, con mayor intensidar y con mayor frecuencia. Impresionantes y de dificil reparación suelen ser las pérdidas de peso que se observan en las rino-faringitis de los neutópatas y de todos los lactantes que retienen agua sin fijarla, o sea, de los que presentan una hidro!abilidad constitucional o adquirida.

Aunque, como ya lo manifestamos anteriormente, son inuchos los lactantes que soportan una rino-faringitis sin que se afecte en forma apreciable el estado general, ánimo, apetito, tre, existe, en cambio, un porcentaje muy alto de ellos en los que es posib': comprobar alteraciones nás o menos profindas en el aspecto, en las actitudes $y$ en las reacciones de los distintos sistemas. Llama la atención, desde luego, que muchos 
niños se tornan pálidos y ojerosos desde los comienzos de tsta afección catarral y no rata vez, desde algunos dias antes de la iniciación de la enfermedad y a semejanza de to que sucede con las bajas de peso. Todo puede quedar ahi y e: enfermo recupera sus colores poroc días después en la convalecencia: pero hay otrcs en que la coloración va rápidamente bacia un tinte tetroso, amarillento o ligeramente cianótico de la piel. Mientras más pequeño o más distrófico es el lactante. tanto más bruscamente puede establecerse este cuadro: en el prematurc y en é diatésico pueden observarse con cienta frecucncia reacciones parecidas. Sin embargo, en nuestro material se encuentran a menudo pequeños enfermos que atraviesan todo el estado agudo, sobre todo cuardo es febril, con una rubicundez muy marcada de la piel de las mejillas, para ponerse Fálíos inmediatamente después de la defervescencia y mant ner esta palidez durante dias y a veces semanas. Cambios de coloración bruscos y repetidos se svelen ver en los neurópatas y exudativos; los periodos de pilidez suelen set impresionantes en estos niños, cuya carita desancajada y afligida suele recotdar la de una toxicosis o de un cuadro abdominal agudo. Fs. las alternativas espontáneas en el aspecto son, en general, de pronóstico grave, sobre todo en los prematuros de poca edac. Advertiremos que al hacer este arálisis hemos descartado hasia donde esto es posible, toda concomitancia del sindroma rinofaríngeo con alguno de los trastcrnos nutritivos agudos que pueden acompañarlo.

La intranquilidad y el llanto que acompañan general. mente a la rino-faringitis suelen ser muy poco apreciables antes del segundo semestre, salvo en aquellos casos en que la afección se extiende al oído medio o que se compruebe un compromiso inflamatorio intenso de la mucosa buco-faríngea. Sin embargo, hay lactantes y se les ve aun entre los que se alimentan al seno exclusivo, que er las primeras semanas y meses de la vida manifiestan una gran agitación y un malestar interso, cada vaz que presentan un estado febril provocado Ficr una rino-faringitís. El llanto de estos niños es desesp:rante, el movimiento de la cabeza, brazos y piernas es permanente, se retuercen como si sufrieran de un gran dolor y sólo descansan por pequeños períodos, después de les cuales vuelve a establecerse el cuadro anterior con igual o mayor intensidad. En algunos de estos enfermos se observan deposiciones frecuentes, pequeñas y ruidosas, sin que existan otros síntomas que justifiquen el diagnóstico de un trastorno nutritivo agudo. Ia alimentación se dificulta y se interrumpe muchas veces 
un el dia por estas crisis de agitación. Para que se produzcan situaciones como la expuesta, es evidente que debe existir una teurofatía, un sistema nervioso excepcionalmente lábil o irritable; pero no porque se haya hecho el diagnóstico de ese estado constitucional, puede dejar de pesquisarse, como suczde tânlas veces. la causa provocadera inmediata de sus manifestaciones, que muy a menudo puede ubicarse en las vías respiratori:s superiores y sin que haya un compromiso ótico u otra localización dolorosa. El valor del riagnóstico precoz se aprecia cuando se ha visto como sufren fstos enfermos aurante 8 ó más dias, mientras se busca la calsa de su mal. y como se mejcran a veces de un día al otro. con un simple tratamiento dercongestionante de la mucosa nasal o una evacuación repitida de las secreciones nasales.

Otra forma de reacción dẹl niño pequeño, tan $c$ más alarmante que aquélla, es el decaimiento y la apatía. La encon. tramos con relativa frecuencia y puede llegar a una atonia total, falta de fuerza hasta para el lianto y para la deglución: la mímica puede desaparecer por rompleto y sólo por momentos se entreabren los párpados con una expresión de vaguedad y de agotamiento profundo en 10 ojos. El cuadro es muy semejante al que presentan las neumenias de forma atónica, descritas por Nassau y sus colaboraderes.

En general, la çituaciớn que se presenta del segundo sementre en adelante, es muy divissa. En esta época e! niño manifiesta su malestar corrientemente por medio del lianto, la intranquilidad, falta de sueño y rechazo de la alimentación. Esto explica. hasta cierto punto, porque se ha arraigado $v$ se manticne todavia el concepto tradicional y rutisario de la "dentición" como causa de esta sintomatología, cada vez que clla se presenta sin que la acompiñan sintomas locales muy riciltantes. Fenemos a la vista algunas curvas de tiemio 2.trás. en las que no se encuentra. frente a ciertas alzas febriles de esta época, otra anotación causal cue "dentición" ? "dispepsia" $y$ : sin embargo, ella va seguida en el transcurso de lcs días contiguos en muchas cbservaciones de la comprokación de una rino-faringitis. de noa bronquitis o de una otiîs. La inspección cuidadosa de ces enfermitos nos ha enseñ?de, en cambio, que la mayoría de las veces no se deja de cen. probar en esos casos una rino-faringitis, leve las primeras veces (n su sintomatología directa y de mayor intensidad y extertsión. cuande las repeticiones han sido frecuentes y su acción distrofiante, más aparente. Controlados, además, sistemáticamente por el oto-rino-laringólogo, estos niños revelan en uir 
porcentaje muy alto, síntomas de un compromiso ótico, los cuales regresan paralelamente a la inflamación rino-faríngea $y$ con mucha frecuencia, sin que haya habido necesidad de recurrir a una paracentesis. Por lo demás, si se considera que la inspección meticulosa de la nariz y de la faringe se descuidaba y se descuida generalmente y que estos lactantes pueden presentar una diarrea o una dispepsia franca, se comprenderá por que un cierto número de los incidentes mencionados figura con al diagnóstico simple de "dispepsia", ignorándose su punto de partida respiratorio. Esto resulta más explicable aun, si se toma en cuenta que muy a menudo la fiebre da entos catarios rino-faringeos no alcanza a persistir más que durante las primeras $24-48$ horas y que aparece entonces como normalizada bajo la acción de la dieta hídrica, que se prescribe como de rigor en esas circunstancias.

Cuando expusimos los síntomas locales de la rino-faringitis, decíamos que la obstrucción nasal y el dolor a la deglución provocaban con suma frecuencia dificultades en la alimentación y que éstas podían llegar hasta la incapacidad totaI para realizar la mamada. Al lado de ellas y dependiente, sin duda alguna, de una reacción especial del sistema nervioso. se manifiesta la inapetencia, que puede ser el síntoma inicial de la infermedad, que puede acompañarla durante toda la evolución o que aparece al final de ella o luego después de la defervescencia. Esta falta del apetito, que muchas veces se suma a las dificultades de origen obstructivo. es en el niño alimentado ai scno, una de las causas más comunes para la interrupción de la lactancia, cuando no se prevé a tiempo y no se combate con paciencia. Cuande en estas circurstancias el hijo no quier? o no puede mamar, depende del tientpo que dute la falta de es timulo de la glándula mamaria, el.que sz establezca en la ma. dre una hipo $\mathrm{c}$ agaláctea. La lactancia puede restablecerse, si la neuropatía no es demasiado rebelde. Frente a sus manifasta. ciones extremas, muchas veces no aueda otro remedio que re currir a la alimentación con leche ordeñada. a la alimentación mixta o artificial y aun a los alimentos concentrados. En los niños que ya están con régimen artificial, suelen presentaros rituaciones semejantes, que oblignn a fraccionar o a concentrer la alimentación. La inapetrencia, el negativismo de estos enfermos puede llegar a un grado tal que es necesario someterse al capricho invencible de ellos, instituyendo regímenes unilaterales y hasta irracionales. con el único objeto de evitar una inanición demasiado prolongada. Esto puede hacerse ata mayor riesgo, siempre que se tenga presente que es inetudib! 
volver al régimen normal lo antes posible. Las distrofias o carencias que se producen cuando no se procede en esta forma, ponen en grave peligro la vida de restos niñcs y terminan casi siempre con una inapetencia crónica y rebelde que va derecho a un estado de hambre y a la descomposición. La disergia y las deficiencias nutritivas se aumentan recíprocamente y el circulo vicioso que se forma, pone a prueba todos nuestros recursos terapéuticos y dietéticos.

Si bien es cierto que gran parte de la sintomatología descrita es la expresión de una reacción especial del sietema nervioso estral y del ccmplejo vago-simpático, no es menos efectivo que en ninguno de los enfermos que hemos tenido a la vista para describirla. existe una base objetiva lo suficientemente clara, para establecer el diagnóstico de un compromiso inflamatcrio del encéfalo o de las meninges. A hacer el análisis de la obstrucción nasal y de sus consecuencias, señalamos entre éstas el opistotono, la agitación, la apatía, el meteorismo y las alceraciones cardio-vasculares y respiratorias. como las más frecuentes: pero dejamos constancia también, que sólo excepcionalmente habíamos encontrado en el líquido céfalo-raquídeo las alteraciones correspondientes a una meningitis sero. sa. $Y$, sin embargo, en las autopsias que se han podido hacer de lactantes fallecidos durante uno de los accidentes respiratorics, hemos comprobado casi siempre una intensa congestión y edema del cerebro y un aplanamiento más o menos acentua. do de las circunvoluciones. No podemos negar, además, que la inmensa mayoría de las meningitis supuradas que se ven en el lactante, revelan como sindroma premonitor de ellas una inflamación del rino-farinx, a tal punte que hasta para la meningitis meningocócica ciertos autores han quetido establecé un sindrema rino-faríngeo con caractetes específicos. Por lo demás, en algunas de nuestras observaciones hemos visto que junto a algunos o al conjunto de los síntomas descritos, los enfermos presentan nistagmus, estrabismo, fibrilaciones de ics músculos de la cara, movimientos de los labios (con propulsión rítmica de la lengua, boca de pescado, etc.), convulsiones generalizadas o de uno o más miembros y estados contracturales parciales o totales pasajeros. El líquido céfalo-raquídeo es generalmente normal o revela alteraciones químico-ci. tológicas, que no alcanzan a salir de lo normal. Este cuadro se insinúa apenas en las primeras $24-48$ horas, se intensifica y generaliza paulatinamente después, para decrecer y desaparecer entre los 6-10 dias siguientes, cuando no ha producido el desenlace fatal durante uno de los ataques convulsivos, que 
van acompañados, la mayor parte de las veces, de apnea $y$ cianosis. Este sindroma de una encefaitis, evidente para nosotros, no es muy frecuente y constituye en realidad una complicación dela rino-faringitis, semejante a la meningitis aguda que también suele cbservatse en el transcurso de ésta. Esta encefalitis se diferencia fundamentalmente, tanto por el comienzo insidicso. la sintomatología y la evolución, de los sindromas convulsivos que se observan en algunos !actantes $y$ niños cada vez que sufren una infección o un alza térmica brusca. Estos sobrevienen repentiramente y los hemos visto tepetirse varias veces junto a cada nueva rino-faringitis simple o complicada. En cierto número de estes enfermos se han comprobado los signos de una espasmofilia: perəen otros faltan per completo. En estos últimos podría pensarse en ana irritación encefálica, producida por una acción toxi-infecciosa fugaz, ya que se trata siempre de accidentes de una duración relativamente corta. Aunque éstas son complicaciones, las mencicnamos aquí, porque muchos de los sintomas generaié expuestos podrian interpretarse como manifestaciones de una encefalitis frustra y que no alcanz: a dar más que una sinto. matolcgía incipiente e incompleta. Esto se bace más plausible si se tiene en cuenta que hoy en dia se tiende a aceptar que muchas de estas infecciones gripates setían causadas por virus y que la flora microbiana que se encuentra en el tino-farinx actuaría sólo secundariamente (Dochez y otros).

Como ya lo dijimos, hay cricnes sostienen que la rinofaringitis no desempeña ningún ró in la génesis de los trastorncs nutritivos agudos y crónicos. Sin embargo. los que así piensan, aceptan uñ causa pura-enteral (bronconeumonía, pielo-nefritis, otitis, etc.) de la dispepsia o de la toxicosis, por ejemplo. Si esas localizaciones infecciosas son capaces de producir una alteración metabólica semejanie, ¿por qué no ha de serlo la rino-faringitis, que al fin de cuentas no es ni más ni menos que una infección e inflamación localizada a un área más o menos extensa de la mucosa respiratoria y que puede comprometer, como lo heros visto ya, en forma grave todos los demás sistemas del pequeño crganismo? En el niño más grande $y$ en el adulto se trata generalmente de una afrción netamente local, sin repercusión apreciable sobre el estado general, y en el lactante suele suceder to mismo: pero en nuectras observaciones vemos que es mucho más frecuente que el catarro rino-faxíngeo vaya acompañado de vómitos, diarrea, baja de peso, sintomas tóxicos, etc., o sea, de un conjunto ncsológico que estamos habituados a interpretar como 
un trastorno nutritivo agudo. Por otra parte, nuestras curvas demuestran que la tepetición o mantención de la infección dz la parte superior del árbol respizatcrio termina tarde o temtrano en una distrofia y a veces elu una descomposición. No discutimos que hay muchas dispepsias, toxicosis y distrofias que nada tienen que ver con una rino-faringitis u orra de las infecciones para-enterales; pero repetimos que nuestra estadítica comprueba que los trastornos nutritivos puros constituyen en los últimos años una minoría pequeña frente a los cus rzconocen como causa una infección extraintestinal y soExc todo, del árbol respiratorio. A nuestra manera de ver las conclusiones erradas se producen y se mantienen por desconocimiento del sindroma causal, que es de difícil ubicación cuando no se le busca especialmente, y porque en este caso se toma generalmente como causa lo que no es más que una consecuencia, es decir, que la rino-faringitis no se diagnostica todas las veces que existe y cuando se diagnostica, esto sucede tardiamente, en plena reparación del trastorno nutritivo, el cual aparece entonces como determinante de la aparición de aquuélla.

Trataremos de analizar este problema de tan alto interés práctico con una revisión de nuestro abundante material, tomándolo desde los trastornos metabólicos más simples o parciales hasta los más complejos. Desde luego, observamos que se nos presentan recién nacidos. prematuros o no, que maman en perfectas condiciones durante los 8-20 días primeros, que a esa altura hacen su primera infección rino-faríngea con o sin temperaturas y que ennjuntamente con ella decaen bruscamente, que rechazan en seguida toda alimentación $y$ que presentan la coloración pálida. terrosa y a v'eces, cianćtica, ya descrita. Agregado a ese cliadro se observa una muçosa bucal intensamente roja. lengua seca con papilas prominentes - lisa, con aspecto de cartae cocida y ligeras manchas de algorra, sin que extetiormente pucdan comprobarse signos de una deshidratación manifiesta. Sin embirgo, para corregir cste cuadro tan alarmante, basta generalmente una hipodermo o fleboclisis con sueto físiológico. toic o con suero glucoadc, con suero de Ringer o de Hartmann, etc. Nosotros preferimos y nos ha dado siempre buen resultado, la ingestión forzada de agua ligeramente azucarada, agua de té o agua con suero de Ringer. Con ello basta para producir en la mayoría de los casos un cambio favorable tan brusco y rápido del aspecto de gravedad que presentan esos enfirmos, que se llega a dudar muchas veces de la justificación de los te- 
mores que habian asaltado al médico. En general y ayudados algunas veces con una o más transfusiones sanguíneas. pasamos en estas circunstancias, de la hidratación inicial, que es siempre de pocas horas de duración. y sin transición, a la alimentación completa, que $d$ niño tenía antes de esta. blecerse este cuadro y que casi siempre es todavía el seno exclusivo. Este sindroma, que se nos presenta la mayoría de las veces anteg del mes de edad, ha sido interpretado por nosotros como la consecuencia exclusiva de una falla en el metabolismo del agua, primer escalón de un trastorno nutritivo agudo de mayor trascendencia. El resultado casi inmediato de la hidratación forzada así parece demostrarlo y la tolerancia por una realimentación completa tan perfecta, prueba que aun no ha alcanzado a producirse una alteración metabólica de tal alcance que pueda ser catalogada entre los trastornos nutritivos agudos corrientes.

Un cuadro semejante, aunque no tan brusco, agudo y grave genaralmente en sus comienzos, lo vemos también en el lactante y sobre todo, en el prematuro, cuando ya están más allá del primer mes de edad. Lo presenta tanto el niño a alimentación materna exclusiva, como el que está alimentado artificialmente; puede acompañar la rino-faringitis del eutrófico y del distrófico y se observa, aunque con menos frecuencia, también en el pre-escolar. De acuerdo con los trabajos de L. F. Meyer, Nassau, Jahr y Kahn, etc., tanto éste como El sindroma anteriormente descritc para el recién nacido, cae en el grupo de los trastornos que hemos designado como deshidratación celular interna en las infecciones agudas del lac. tante y del pre-escolar (Schwarzenberg, Arch. Latino-americanos de Pediatría; tomo XXI, N. ${ }^{\circ}$. año 1927). Descrito $E n$ detalle y discutido ampliamente en aquella oportunidad, nos ahorraremos una nueva insistencia sobre sus peculiaridades y su significado. Sólo diremos que para salvar este c.scl'c, hemos recurrido a la dieta hiperazucarada, haciendo como en el caso anterior una vielta rápida a la alimentación natural, cuando no existían signos de un trastorno nutritivo más avanzado y cuando ya el sindroma habia cedido ( 2 ó más dias, con un máximo de 51 . Los resultados han sido siempre halagadores y en todo semejantes a los que presentamos en el trabajo de nuestra referencia. Estimamos que cualesquiera de las dietas hidrocarburadas, llamadas por algunes hidratantes y tan en boga en la actualidad, puede ser tan benéfica como aquélla; tan es así, que en estas circunstancias las usamcs indistintamente. Volvemos a repetir, el 
diagnóstico de la infección rino-fatíngea, discutido casi siempre cuando la inflamación local no es muy aparente en estos enfermos. se confirma, a pesar do todo, por la aparición de los síntomas locales después del accidente, por la comprobación de la lesión concomitante del oído por parte del oto-xinolaringólogo o por la coincidencia de otras infecciones gripales en la familia o en el servicio a que pirtenece el enfermo. Suelen coexistir, además, sintomar bronquiales fugaces y de poca extensión.

El trastorno nutritivo que arompaña ia rino-faringitis Fuede quedar circunscrito, tal como pasa con cualquiera otra infección aguda en esta época de la vida, a esa alteración exclusiva del metabolismo del agus. que hemos designado como deshidratación celular interna. Ella puede regresar como lo hemos expuesto; pero puede ser la iniciación de un trastono más amplio o presentarse conjuntamente con toda la sintomatologia de éste. Efectivamente, en nuestras observaciones, encontramos muy a menudo, tanto en el comienzo como en el transcurso de la rino-faringitis, la dispepsia franca, el enterocatarro y la toxicosis. Importa $p$ interesa en estos casos que se establezca si el origen de estos tristornos nutritives agudos es para-enteral o nutritivo puro y para la primera de estas posibilidades, a qué localización infecciosa extra-intestinal corresponde la causa de ellos. La infección para-enteral puede sospecharse en los primeros dís y cuando aun no han podido comprobarse los síntomas locales de la rinofaringitis, por el hecho que la dieta hídrica no ha sido capaz de normzlizar la temperatura. Indicio de que existe una infección o que, por lo menos, prevalece un factor infeccioso sobre el metabólico o nutritivo, es, además, según nuestra experiencia. l’ coexistencia del sindroma de dechidratación celular interna $y$ sobre todo de su componente escleredema. Posteriormente es posible, casí siempre, corroborar el diagnóstico por la comprobación de los sintomas locales directos e indirectos o desecharlo ante la evidencia de cualquiera otra infección. sea intestinal o para-enteral. El valor del diagnóstico causal reside en que establecido el origen para-enteral de estos trastornos. cri muchos enfermos, sobre todo después del segundo semestre de edad, las medidas dietéticas pueden ser de aplicación breve y restringida y que al cabo de pocos días esté restablecido el régimen normal de alimentación. Tenemos un sinnúmero de dispepsias y aun de entero-catarros provocadas por un catarro rino-faringeo, en las que no hemos tenido necesidad de recurrir a prescripciones dietéticas especiales: ha bas- 
tado con dejar que el enfermo restrinja durante uno o varios días su alimentación ad libitum que reemplace lo que deja de ingerir por cantidades suficientes de líquido. En estos casos, la intolerancia gástrica, la ciarrea y la curva de peso se normalizan paralelamente a la ateruación de los síntomas inferciosos y al restablecimiento del apetito. Como comproEación casi experimental de lo dicho. estamos en situación de Fresentar algunas curvas de niñor que han tenido una rinotaringitis repetida, en los cuales frente a unc de sus cuadros infecciosos acompañados de una dispepsia, se instituyó el régimen dietético pertinente, de corta duración y con buen resultado; 15 ó más dias después y frente a una situación exactamente igual se dejó que $e^{1}$ riño restringiera su alimentación, se reémplazó el déficit cot agua y se obturo el mismo resultado, con una reparación máx corta. Esto demosiraría, a nuestro juicio, que aunque se presenten deposiciones alteradas $y$ frecuentes, uno que otro vómitc y una baja de peso, el irastorno nutritivo no ha alcanzado en esas condiciones la alteración funcional suficiente para que se justifique la prescripción de una dieta hídrica y la realimentación progresiva, que si se repite con intervalos muy cortos, no es indiferente.

Por lo demás, ya Finkelstein hablaba de las diarreas irritativas, producidas por vía simpático-parasimpática (acción de toxinas) y que pueden observarse en cualquiera inlección paraenteral, sin que se logre establecer un trastorno nutritivo propiamente tal. Por otra parte, ya citamos el caso de los lactarites alimentados al seno exclusivo, en los que la 'ino-faringitis puede ir acompañada de deposiciones frecuentes, desagregadias, mucosas, etc.. que se normalizan también, sin que tengamos necesidad de intervenir en nada para corregirlas aunque se hayan manifestedo conjuntamente con uno que otro vómito y una detención o baja de la curva de peso. $\mathrm{Si}$ en estos enfermos se forma, como se ve muy a menudo. un eritema peri-anal más o menos intenso y si esta inflamación compromete el esfínter, lo scbrepasa e invade una parte de la mucosa del recto, puede aprecer un tenesmo más o menos acentuado, el número de deposiciones se eleva y éstas toman un carácter mucoso o muco-sanguinolento. Ante esta sintomatología se impone para muchos el diagnóstico de entero-colitis $y$ se instituye la terapéutica correspondiente, tanto más si no se ha hecho el diagnóstico de la rino-faringitic o de otra infección para-enteral. Sin embargo, tenemos * la vista algunas observaciones de lactantes. en los cuales esta sintomatología desaparece con la mejcria del catarro respira- 
torio y sin que se hubiera prescrite ninguna medida dietética especial. Hemos hecho el análisis extenso de todos estos fenómenos con el único objeto de recalcar cuán dificil se hace muchas veces en estas circunstancias establecer cuándo y cómo sie inicia realmente un trastorno nutritivo agudo o una complicación intestinal, en qué condiciones debe hacerse el trat:miento pertínente y cuándo puede evitarse un régimen de restricción alimenticia.

Cuando estos trastornos nutritivos agudos se presentan con el catarto respiratorjo del distrófico, diatésico o prematuro, sus manifestaciones $y$ sus consecuencias son mucho más graves. Ya en ellos no es posible mirarlos como aferciones secundarias y sin trascendencia, como puede haceriz en muchos de los casos anteriores, porque el daño de la pared intestinal es aquí generalmente más intenso y más extenso y la alteración resultante del quimísmo intestinal y del metabolismo es mucho más profunda también. Las consecuencias graves de esta situación se ven en muchas curvas en más de alguna oportunidad y casi, sin exrepción. todas las veces aue la rinc-faringitis se acompaña de un trastorno nutritivo agudo. La gravedad que reviste la repercusión de una infección como la rino-faringitis sobre el estado general, se nos revela cuando ésta se injerta en el periodo de reparación de un trastorno nutritivo agudo o de una distrofia $y$ cuando i: comprueba, como lo observamos con frecuencia. que al alimento tolerado hasta ese momento en perfectas condiciones, actúa de ahí en adelante como un verdadero venen? Czerny, Keller, Finkelstein, etc.). El máximo de frapilidid en este sentido lo vemos en los lectantes que ban sido alimentados con un exceso de harinas, er los hidrolábiles y en los neurópatas y exudativos.

Para una mejor comprensión y explicación de muchos de los sintomas generales anterictmente expuestos y de algunas de sus formas. nos parece do interés recalcar que en numerosas autopsias de lactantes fallecidos dentro de las primeras 24.48 horas de enfermedad, a causa de una de las infocciones llamadas hiperagudas o hipertóxicas, hemos comprotado alteraciones especificas de la mucosa intestinal y de ctros órganos, semejantes a las que se observan en la inhibición capilar, en el shock. En algunas de ellas, la única localización inflamatoria o infeccios: que pudo establecerse, se encontraba en el rino-farinx. Si esta alteración se presenta en forma indiscutible en los casos de esas infecciones foudrsyantes nos parece que no es descabellado pensar que puedan 
Existir lesiones semejantes, aunque de menor intensidad, en el periodo inicial o de invasión de cualquier estado infeccioso y también de la rino-faringitis (en su carácter de infección general en el lactante) y a pesar que su transcurso posterior se haga en forma menos brusca, aguda y dramática. Las manifestaciones a distancia $y$ el compromiso del estado genera: podrían explicarse a base de estos hechcs por la participación vago-simpática que seguramente genera o exacerba muchos dé los síntomas descritos, tan es así que hasta las llamadis por Finkelstein diarreas irritativas (entero-colitis secundarias) se producen para él por esta vía. Es lógicc suponer, эđ̊̊más. que tales repercusiones sean más frecuentes, más evidentes y de mayor trascendercia en los sujetos afectos a una labilidad especial de su sistema nervioso, como son los prematuros, diatésicos y algunos distróficos. por ejenpio. En algunas de nuestras observaciones se ve. por último. quc esta sensibilidad exagerada puede ser adquirida también y manifestarse solamente después que el niño ha sufrido varias: invasiones infecciosas de este tipo.

Si todo lo dicho anteriormente vale para los trastornos nutritivos agudos, que una simple rino-faringitis puede desencadenar en el lactante, no es menos evidente que puede ser ella también, tal como lo hace ror lo demás, cualquiera ctra infección, la que lo lleva paulacinamente a una distrofia y hasta a la descomposición. Este hecho se percibe sin lugar a dudas cuando la inflamación naso-faríngea se repite periódicamente o se mantiene durante un tiempo más o menos lar. go tal como se observa en numetosas de nuestras curvas, en las cuales la distrofia es la consecuencia de días y semanas de inapetencia. de intolerancia por el alimento y de dificultade: mecánicas en la alimentación, que de tiempo en tiempo reaparecen y caen con todo el peso de la insuficiencia nutritiva prolongada sobre el organismo infantil. Este aspecto particulat $10 \mathrm{da}$ especialmente la forma recidivante de la rino-fariñ gitis, que se encuentra sobre todo en el primer año de la vida: perc s: ve también en aquella forma, que ya describimos, $y$ que aun en el lactante alimentado al sero exclusivo, man. tiene durante un largo tiempo la inflamación local, una temceratura subfebril y a veces más alta y todos los trastornos de carácter general de que hemos hablado $y$ que sin transición provienen directamente del estado agudo y lo prolongan. La acción distrofiante de esta infección subaguda reciVivante o mantenida salta aun más a la vista y llega a $r=r$ imptesionante, cuando en el transcurso de ella se comprueban 
las agravaciones que produce cualquiera de las complicaciones de la rino-faringitis (otitis, bronquitis, etc.) y que figuran frecuentemente como causa determinante de los trastornos in tritivos agudos, que se observan simultáneamente. Comúnmente son ellas las que aparecen en la clínica y son ellas las que determinan la etiqueta de estos accidentes, cuando en cealidad no son más que brotes incidentales de la invasión o extensión de la infección que se mantiene $y$ que puede comprobarse en el rino-farinx. Esto se demuestra, sin lugar 2. dudas, cuando el examen local se hace con cuidado y en lo forible diariamente: otra prueba evidente la dan la persistencia y la exaccrbarión de los infartos ganglionares correspondientes, ccmo puede verse en muchas de nuestras observacio. ras. Por otra parte, corrobora este aserto el que nuestro oto‘ino-latíngólogo, solicitado para e! examen repetide de estos niños y sim adverencia previa. haya comprobado la mayoría de las veces. una sintomatología local identica a la que nosctros habiamos anotado. Por las razones que ya hemos expuesto antes, no puede extrañar cue el diagnóstico clinico se haya hecho en algunos casos con dos a seis días de antelación al del especialista; esta divergencia ha sido tanto más frecuente, cuanto menor era el lactante y cuanto mayor era su distrofia y su prematuridad. Lo importante para nosotros está en que, a pesat de estas divergenciar más aparentes que reales. a la postre se llegara siempre a un diagnóstíco concordante $y$ sin que se hubiera trabajado de acuerdo o con consulta previa. Si esta cooperación se llevara a cabo sistemáticamente en los servicios de lactantes y con mayor frecuencia, el porcentaje de los diagnósticos de la rino-faringitis se elevaría en forma notable y sus modalidades, sus complicaciones $y$ sus consecuencias sobre el organismo infantil, serian seguramente más conocidas.

La interptetación de esta sintomatología sombria y comFícada. que puede presentarse er la iniciación o en e] transcurco de una rino-faringitis, suelp bacerse aún más difícil. cuando se agrega a ella en forma aguda o subrepticía una de las infecciones intestinales del tipo de las shigellosis, s7lmn. nellosis u otras. En algunos casos y llama la atención aue muchos de ellos correspondan a tiños eutróficos, hemos cbservado que después de dos o más dias de evolución de in rindroma naso-faríngeo, se instala bruscamente un cuadro de cntero-cclitis, acompañado de una elevación notoria de la temperatuta y de manifestaciones toxicas intensas. En otros y más frecuentemente en lactante. distróficos, esta entero- 
clitis aparece en forma insidiosa y llega a su culminación en $e$. lapso de algunos dias. La comprobación bacteriológic: es posible en la mayoría de los casgs y corresponde siempre a niños sometidos a una alimentición artificial $o$, por 10 menos. mixta. La mayor incidencia de esta infección agre. zada la encontramos en los meses de primavera y verano. En las autopsias que se han podido hacer se comprueba poi un lado el compromiso del árbol respitatorio superior. con cxtensión al oído, etmoides o pulmón, y por el otro, las leciones específicas de una entero-colitis aguda. Entre los niños que han llegado a la mesi de necropsia se encuentran ics que han fallacido a las 24-48 horas después de la instauración del cuadro intestinal y los distróficos, que después de d'a han ido rápidamente a la descomposición.

(Continuará). 